\title{
Die Entstehung der Städte des Erzstifts Köln am Niederrhein ${ }^{1}$ ). \\ Von
}

Th. Ilgen.

Die Städte des Niederrheins, insbesondere die des Erzstifts Köln, sind bei der allgemeinen Diskussion über die Entstehung des Städtewesens bisher recht schlecht weggekommen. Nur gelegentlich einmal begegnet man in den Erörterungen über diesen oder jenen Punkt des Ursprungs der Städte und der Entwicklung der Stadtverfassung den Namen Andernach, Siegburg, Wesel oder Rees. Die überwiegende Bedeutung Kölns hat nicht nur im Mittelalter die Städte am Niederrhein nicht zu besonderer Machtentfaltung kommen lassen, das Alter und die Fülle des für Köln vorlandenen archivalischen Materials hat auch die Aufmerksamkeit der neueren Geschichtsschreiber von der Beschäftigung mit der Geschichte der ubrigen Städte des Gebietes abgelenkt. Dabei hat freilich auch mitgewirkt, dass die Quellen zur Rechts- und Wirthschaftsgeschichte der niederrheinischen Städte weder für einzelne Orte, noch für grössere Gruppen von solchen in übersichtlich geordneten Drucken vorliegen. Ferner scheint die Vielgestaltigkeit der städtischen

1) Die nachfulgende Uebersicht soll eine gedrangte Zusammenstellung der Städte des Gebietes im Mittelalter geben und die für deren Entstehung bedeutsamsten Merkmale kurz herausheben. Sie ist durch Vorarbeiten veranlasst, welche die von der Gesellschaft für Rheinische Geschichtskunde geplante Herausgabe der Quellen zur Rechts- und Wirthschaftsgeschichte der Städte des Niederrheins nothwendig machte. Die gelegentliche Ausbeute an bisher nicht veröffentlichtem archivaltschen Material wird nur Stichproben verdankt, welche ich an mir leicht zuganglichen Stellen angestellt habe. Vollständigkeit in der Heranziehung. der gedruckten Litteratur war ebenfalls nicht beabsichtigt.

Annalen des hist. Vereins LXXIV. 
Gebilde, welche man für eine Eigenthümlichkeit der niederrheinischen Lande erklart bat ${ }^{1}$, die Forscher, welche sich mit den Fragen der Städteentwickelung im Allgemeinen befasst haben, von der Berücksichtigung der Städte dieser Gegend abgehalten zu haben. Doch vielleicht stellt sich bei näherem Zusehen auch dieser Zustand nicht so mannigfaltig heraus, wie man ibn einstweilen noch annimmt. Haben doch schon die kurzen Zusammenstellungen, welche G. von Below in seiner landständischen Verfassung in Jülich und Berg über deren Städte veröffentlicht hat ${ }^{2}$ ) und Liesegangs Arbeit über Niederrheinisches Städtewesen vornehmlich im Mittelalter ${ }^{3}$ ), die sich mit den clevischen Städten befasst, gezeigt, dass sich zum mindesten unter den Städten desselben Territoriums gleichmässige Phasen der Entwicklung aufdecken lassen. Man wird noch weitere verwandte Bezüge herausfinden, wenn man die Städte ihrer Entstehungszeit entsprechend zusammenstellt. Das dürfte schon die kurze Uebersicht über die Städte des Erzstifts Köln, die wir zur Ergänzung der Arbeiten G. von Belows und Liesegangs nachfolgend liefern, mit aller Deutlichkeit zeigen.

Den Blick von den niederrheinischen Städten - Köln selbstverständlich auch hierin wieder ausgenommen -- al)gezogen hat aber auch die Erwägung, dass man sich sagte, hier ist das eigentliche städtische Verwaltungsinstitut, der Rath, durchgängig in Anlehnung an die Gerichtsbehörden, die Schöffenkollegien, entstanden, für die Ausbildung der besonderen Stadtverfassung vermag daher diese Gegend keine schlagenden Beispiele zu liefern. Dieser Satz ist richtig, die Schaffung eines besonders abgegrenzten und in bestimmter Weise privilegirten Gerichtsbezirkes mit eignen Urtheilsfindern macht in erster Linie das Wesen der niederrheinischen Stadt aus, das ist der Rahmen, in dem sich das eigentliche Städtebild entwickelt.

Als sich von der zweiten Hälfte des 11. Jahrhunderts $a b$ neben Köln Städte, Oppida, Civitates liberae, am Niederrhein neu bildeten, war eine das ganze Gebiet umspannende Organisation der Landgerichte hier vorhanden. In diese sind auch die alten

1) Vgl. L. Korth in dieser Zeitschrift $62 \mathrm{~S} .190$.

2) In der Zeitschrift des Bergischen Geschichtsvereins 21 S. 205 ff.

3) In Gierkes Untersuchungen zur deutschen Staats- und Rechtsgeschichte, Heft 52 . 
römischen Civitates und Castra, welche in fränkischer Zeit als Wohnstätten weiter benutzt waren, wie Andernach, Bonn, Deutz, Neuss, Zülpich und Xanten eingegliedert worden. In einigen derselben werden wir wohl Gauvororte zu erkennen haben, an welchen das Hauptgericht des Grafschaftsgebietes abgehalten wurde. Und ebenso fanden die kirchlichen Centralstellen, die späteren Dekanate, von denen aus die Christianisirung der Umgegend geleitet wurde, hier ihren Sitz. Gewöhnlich sind es zunächst Ordensniederlassungen, deren Kirchen an den Hauptfesttagen im Jahr der Sammelpunkt aller in den umliegenden Wohnplätzen angesessenen Glänbigen wurden. Auf diese Weise entwickelte sich um die Stiftskirchen ein Verkehr, der durch Marktprivilegien der Kaiser gefördert wurde. Bonn, Siegburg, Rees, Xanten waren, wie uns unsere Urkunden erkennen lassen, Marktorte, ehe sie durch die Ueberlieferung als Städte gekennzeichnet werden.

Zur Klärung unserer Auffassung von der Entwicklung der einzelnen Niederlassung zur Stadt wird es unbedingt beitragen, wenn wir nachweisen können, in welcher Weise der Ort aus dem bisherigen Gerichtssystem herausgeschält und als Stadtgericht besonders organisirt wurde. Das vermögen wir bei Siegburg durch verhältnissmässig recht alte Urkunden, die uns zeigen, wie das städtische Gericht eingerichtet worden ist und schrittweise an besonderen Privilegien gewonnen hat. Siegburg gehört zwar in späterer Zeit nicht mehr zum Erzbisthum Köln ${ }^{1}$ ), aber es ist doch als eine Gründung des beiligen Anno anzusehen und hat noch durch Erzbischof Plilipp von Heinsberg eine Bestätigung seiner städtischen Rechte erfahren.

An Stelle einer alten Burg auf dem isolirten Bergkegel hat Erzbischof Anno von Köln in den $60 \mathrm{er}$ Jahren des 11. Jahrhunderts ein Benediktinerkloster gestiftet. Bei dieser Gelegenbeit wurde die Villa Antreffa an den Fuss des Berges verlegt. Sie hat offenbar als die Muttergemeinde der späteren Stadt Siegburg zu gelten, obwohl ihr Name verschwunden ist. Da der Abtei durch königliche Gunst ein Markt, der Zoll und eine Münze bewilligt wurde, haben sich schon bald nach deren Gründung zahl-

1) Mit der Reichsunmittelbarkeit haben die Aebte von Siegburg auch die Hoheit über die Stadt erworben, deren sie erst 1676 verlustig gegangen sind. S. Schwaben, Geschichte der Stadt, Festung und Abtei Siegburg S. $92 \mathrm{ff}$. 
reiche Kaufleute als Ansiedler bier eingefunden. Sie nabmen auch Theil an den Vergüustigungen, wélche weiterhin der Stiftung Annos zugewendet wurden. Die Aebte verstanden es im 11. Jahrhundert durchzusetzen, dass der Vogt der Abtei nur einmal in Jahre an drei aufeinander folgenden Tagen für die Leute der Abtei im Umkreis der Siegburg Hochgericht halten sollte, zu dem an je einem Tag ein bestimmter Theil der Bevölkerung zu erscheinen hatte und an dem ïber Fälle von Blutrunst, Diebstahl, Friedbruch und strittiges Erbe geurtheilt wurde ${ }^{x}$ ). Statt der drei ungebotenen Dinge im Jahr wurde also für das Siegburger Gebiet nur eines eingerichtet. Durch König Heinrich IV. erlangte Abt Erpho von Siegburg im Jahre 1071 dann das weitere Privileg, dass ihm der Bann in einem festgesetzten Umkreis um den Berg, unbeschadet jedoch der Gerichtshoheit des Grafen, der mgleich Vogt des Klosters war, zugesprochen wurde mit der Befugniss, von jedem, er sei Höriger oder Freier, die 60 Schillingsbusse zu erheben, der im Bannbezirk einen gefangen nehmen, ausplündern, verletzen oder in sonst einer Weise schädigen würde. Die Massregel war gegen Uebelthäter gerichtet, welche die zum Markt Siegburg ziehenden Kaufleute belästigten und die es verstanden, sich der Sühne gegenüber dem Grafen des Gerichts wie anch der des Herrn der Bedrängten zu entziehen ${ }^{2}$ ). Die Grenzen des Bannes sind genau umschrieben, sie entsprechen dem Umfang des uns aus späteren Zeiten tiberlieferten Burgbannes ${ }^{3}$ ). In diesem behieiten nur die Gerichte der drei Höfe, Sieglar, Geistingen und Oberpleis ihr bisheriges Recht, das von deren Schultheissen ausgeüht wurde ${ }^{4}$. Im Laufe der Zeit hat danu das Privileg von 1071 die Ausdehnung erfahren, dass kein Bürger von Siegburg einer Ladung des Grafen (des Vogtes) an eine andere Gerichtsstelle der Grafschaft zu folgen brauchte und dass alle Klagen der Landsassen resp. der Unterthanen des Grafen gegen Siegburger Bürger vor das Schöfengericht in der Stadt gebracht werden mussten, aus-

1) Ueber die ältesten Siegburger Urkunden und deren spatere Entstehung'szeit hat neuerdings o. Oppermann eingehende Untersuchungen angestellt (Westdeutsche Zeitschritt 31, S. 59 ft.), aut die ich hier einfach hinweisen muss.

2) Lacomblet, Ukb. I 214. Vgl. Oppermann a. a. O. S. 86.

3) Vig. diese Zettschrift 23, S. 62 und $126-128$.

4) Op permanua. a (). S. s6 hail dıese dı ei fur Hochgerichtsstatten. 
genommen den Fall, dass der Angeschuldigte das Recht verweigerte. In diesem Sinne hat Erzbischof Philipp von Köln im Jahre 1182 die Gerichtsgewalt und das Burgrecht der Stadt Siegburg formulirt ${ }^{1}$ ). Bei dem Alter dieses Zeugnisses, das bisher keine sonderliche Beachtung gefunden hat, können wir es uns nicht versagen, dieses hier im Wortlaut einzurücken:

"Item judiciaria potestas et ius burgense, quod oppidum Sigebergense eatenus dinoscitur habuisse, secundum tenorem privilegiorum et secundum quod a tempore fundatoris sui beati Annonis predecessoris nostri ei constitit, manebunt ei inconvulse: ita ut nullus burgensis extra in potestatem comitum vel quod vulgo Vestene dicitur vocetur, sed, si quis provincialis vel qui de potestate comitum est adversus burgensem pro violata pace vel pro causa qualibet magna sive modica proponit, iusticia vel lege scabinorum super hoc contentus sit intra civitatem, nisi forte inculpatus iudicio stare contemnat, ut merito de eo ubique locorum dignum sit indicari."

Siegburg wird trotzdem, dass zahlreiche Kaufleute dort angesessen waren, wie wir aus Urkunden des 11. Jahrhunderts entnehmen müssen, im Jabre $112 \tilde{5}$ noch als Villa bezeichnet ${ }^{2}$ ). Dann scheint ihm der Manerring, der wahrscheinlich noch im 12. Jahrhundert um die Stadt gezogen ist, den Namen Oppidum oder Civitas eingetragen zu haben. Innerhalb dieser Befestigung sowohl wie im weiteren Burgbanne galt das oben geschilderte besondere Burgrecht3), das schon früb auch nach der civilrechtlichen Seite seine Ausbildung erfahren hat $\left.{ }^{4}\right)$. Der oberste Grundsatz städtischer Freiheit aber bestand darin, dass der Siegburger Bürger gegen willkürlicbe Belästigung durch ein höheres Strafmaass, das auf diese gesetzt ward, rechtlich besser geschützt blieb, als die ausserhalb des Bannes Angesessenen und dass er das Vorrecht

1) La comblet, Ukb. I 483 2) Lacomblet, Ukb. I 300.

3) So müssen wir den lateinischen Ausdruck ius burgense nach der schon im Mittelalter am Niederrhein üblichen Uebertragung wiedergeben; im deutschen Recht der Stadt Bonn erscheint das Wort burchrecht. S. unten S. 8 Anm. 6.

4) Vgl. die Urkunde Abts Gerlach von Siegburg von 1187 (Lacomblet, Ukb. I 506). Item frater predictus (Bruder Heinrich von Siegburg) ab Heinrico magistro curtis suẹ in Sigeburg emit curtile in eadem civitate pro triginta solidis et adquisivit illud iure burgensi solvens duos solidos annuatim. 
genoss, bei Vergehen seinerseits nur vor dem Schöffengericht in Siegburg abgeurthreilt werden zu können.

Für die Stadt Neuss soll schon Erzbischof Anno im Jahre 1074 ein besonderes Schöffengericht bestellt haben, indem er dazu seinerseits 6 Schöffen ans seinen in der Stadt wohnenden Ministerialen ernannt habe, während er den Bürgern von Neuss ztlgestand 6 Schöffen aus ihrem Kreise zu wählen. Die Crkun(e' ${ }^{1}$ ), welche uns von solchen Einrichtungen Kenntniss giebt, ist zwar gefälscht, ihr Inhalt dürfte aber doch im Grossen und Ganzen für die Neusser Gerichtsverhältnisse des 12. Jahrhunderts zutreffend sein ${ }^{2}$ ). Sicher ist wohl auch, dass Neuss bereits in dieser Zeit befestigt gewesen ist ${ }^{3}$ ). Der Gerichtsbereich der Neusser Schöffen umfasste die Stadt and einen Theil des umliegenden Gebietes, das uns in einer Urkunde von 1254 als Burgbann überliefert ist $\left.^{4}\right)$. Den Vorsitz im Schöffengericht führte im 13. Jahrhundert der erzbischöfliche Schultheiss ${ }^{5}$ ), der wohl die Stelle des ehemaligen Vogtes des Grafengerichts eingenommen hat. Dass die Bürger von Neuss einer Ladung an ein auswärtiges Gericht nicht zu folgen brauchten, erfahren wir durch einen Spruch König Rudolfs vom 4. Dezember $1282^{6}$ ).

Eine bemerkenswerthe Reform des Andernacher Schöffenkollegs ist im Jahre 1171 durch Erzbischof Philipp von Köln ins Leben gerufen worden. In dieser Stadt, welche bereits 11297)

1) Abgedruckt bei Kremer, Akademische Beiträge II p. 203 ff. und bei Lacomblet, Archiv für die Geschichte des Niederrheins II S. 326 ff.; vgl. dazu die Vorbemerkungen Lacomblets S. 319 ff.: Die letzten Spuren des fränkischen Salhofes zu Neuss.

2) Oppermann a. a. O. S. 30 verlegt die Entstehung der Fälschung erst nach 1242, indem er die darin wiedergegebene Zusammensetzung des Schöffenkollegs auf die durch die Reform von 1259 (s. unten) herbeigeführten Zustände bezieht. Vgl. auch Tücking, Geschichte der Stadt Neuss S. 13.

3) Im Jahre 1190 werden burgenses de civitate Nussia erwähnt bei Lacomblet, Ukb. I 524 vgl. auch 539.

4) Lacomblet, Ukb. II 408. Vgl. hierzu Tücking S. 187 ff. insbesondere 194, wo nach älteren Weisthümern die Grenzen des Burgbannes beschrieben sind.

5) S. die Urkunde von 1242 über die Stiftung des Hospitals in Neuss, Lacomblet, Ukb. II 273.

6) S. Tücking a. a. O. S. 30.

7) S. Knipping, Die Regesten der Erzbischöfe von Köln II 248. 
mit Mauern umgeben worden, war es dahin gekommen, dass arme Leute das Schöffenamt versahen, die sich in ihrem Urtheil nicht frei zu halten wussten. Die Ueberlieferung deutet darauf hin, dass sie bislang ein Siebener-Kolleg gebildet hatten. Sie wurden ihres Amtes entsetzt und an ihre Stelle nunmehr die doppelte Zahl, nämlich 14 Schöffen aus den verständigeren, wohlhabenderen und angeseheneren Einwohnern nea gewählt und zwar auf Lebenszeit. Bei ihrer Wahl gelobten diese, sich mit ihren Urtheilen nach den Gewohnheiten der Mutterstadt Köln und der anderen erzbischöflichen Städte richten zu wollen ${ }^{1}$ ). Für die Kenntniss der Kreise, aus welchen das neue Schöffenkolleg genommen war, ist die Bemerkung werthvoll, dass die Rechtsprechung in Andernach dadurch keine Verzögerung erleiden sollte, dass das eine oder indere Mitglied auf Handelsreisen abwesend war. Bei schwereren zı entscheidenden Fällen genügte die Anwesenheit von 7, bei geringeren die von 3-4 Schöffen. Auch von der Busse wegen Nichterscheinens auf dem Grafending wurden die Schöffen bei ausreicbender Entschuldigung befreit. Nach einer Verordnung desselben Erzbischofs Philipp von 1173 durfte der Wechsel in Eigengut nur vor dem Andernacher Schöffengericht erfolgen ${ }^{2}$ ), das aber auch für Veräusserungen von Erbzinsgut zuständig war ${ }^{3}$ ). Der Andernacher Gerichtsbezirk umfasste ausser der Stadt selbst noch Meissenheim, Keel und Namedi. An dem städtischen Regiment in Andernach haben seit alter Zeit neben den Schöffen die dort wohnenden Ritterbürtigen Antheil, zu denen sich am Ende des 13. Jabrhunderts die Majores ${ }^{4}$ ) gesellen, während auch die Gesammtgemeinde um diese Zeit eine Vertretung gefunden haben muss. Erst gegen die Mitte des 14. Jahrhunderts begegnen uns Burger meister und Rath von Andernach5).

1) Beyer, Mittelrheinisches Ukb. II 5. Vyl. dazu Hoeniger, Ursprung der Kölner Stadtverfassung in der Westdeutschen Zeitschrift II S. 242 und 243.

2) Vgl. R. Hoeniger, Der Rotulus der Stadt Andernach in dieser Zeitschrift 42 S. 9.

3) Vgl. ebenda S. $11 \mathrm{Nr} .14$ und öfters.

4) Erwähnt in einer Urkunde vom 3. Mai 1287 bei Gengler, Codex iuris municipalis Germaniae S. 43.

5) Sie erscheinen in einer Urkunde von 1358 (St.-A. Düsseldorf, Kurköln Suppl. Nr. 37) Rittere, scheffene, burgermeistere, raid ind gemeyne burgere zi̊ Andernache. 
Die Drususstadt am Rhein, Bonn, hat ebensowenig wie die anderen römischen Ansiedelungen Spuren ihres Verfassungszustandes aus der Römerzeit in das Mittelalter hinübergerettet. Im Anfang des 11. Jahrhunderts tritt sie uns in einer Urkunde als Villa Bonnensis entgegen ${ }^{1}$ ). Doch schon bald nach dieser Zeit muss sie grössere Bedentung gewonnen baben ${ }^{2}$ ). Indessen Stadt nach der Rechtsauffassung des Mittelalters ist Bonn erst in der ersten Hälfte des 13. Jahrhunderts geworden. Im Jahre 1243 (1244) Mär\% 18. erlaubt Erbischof Conrad von Köln den Rittern, Schöffen und der Bevölkerung von Bonn die Stadt mit Mauern und Gräben zu umgeben und den weiteren Ausbau der städtischen Verfassung nach dem Gutdünken der dortigen Schöffen ${ }^{3}$ ) vorzunehmen. Nicht viel später, im Jahre 125̃5, erfuhr der Vogteibezirk des Bonner Gericlıts eine erhebliche Schmälerung, indem die auf dem anderen Ufer des Rheins gelegenen Theile abgesondert wurden ${ }^{4}$ ). Damals wird der Bonner Stadtbann festgelegt sein, zudem auch die Dörfer Dransdorf und Graurheindorf ober dem Bache gebörten ${ }^{5}$ ). Das war das Gebiet, welches, wie uns eine Aufzeichnung aus dem 14. Jahrhundert überliefert, auf Schöffenurtheil gefreit war ${ }^{6}$ ). Die Bonner Schöffen durften, wenn ein Bürger verwundet oder gar getödtet wurde, innerhalb des Bannes auf Geldstrafe erkennen des Friedens halber, selbst gegen den Willen des Geschädigten. Der Schuldige hatte auf sein $\mathrm{Burgrecht}$ hin 3 Tage Frist, um sich mit seinem Gegner aus-

1) L ac o mblet, Archiv für die Geschichte des Niederrheins II S. 302.

2) Vgl. die Aufsätze Lacomblets, Die römische Basilika zu Bonn und die Hofesverfassung im Bezirke der Stadt Bonn in seinem Archiv II $65-99$ und 296-318.

3) Lacomblet, Ukb. II 284.

4) Lacomblet, Ukb. II 419.

5) Vgl. (Eichh of) Historisch-geographische Beschreibung des Erzstiftes Köln S. 72. Genaue Grenzbeschreibungen aus späteren Jahrhunderten sind in den Akten Kurköln, Stadt Bonn des St.-A. Düssseldorf erhalten.

6) S. die Bonner Stadtrechtsaufzeichnung aus dem 14. Jahrhundert, gedr. Lacomblets Archiv II S. 318: Vort off eynichem unsem ingesessen burgere eynge ungeval gescheege an wunden off an doitslage, want die stadt gevryet is up scheffenurdel, ind der scheffen wyst den zu erdingen up die wunde off up den doiden, dry dage up syn burchrecht ind seehs wechgen up syn lantrecht, dat da enbynmen dryn dagen ind seehs wechgen syn lyff ind guet sal vry șrn ind ungekrut van yeman in rechte. 
Die Entstehung der Städte des Erzstifts Köln am Niederrhein.

einander zu setzen; nach Landrecht war diese auf 6 Wochen bemessen $^{1}$ ).

Das in Bonn gegruindete Cassiusstift besass daselbst für den Tag der Erhebung der Gebeine seiner Stiftsheiligen am 10. Oktober laut dem Privileg Erzbischof Reinalds von Dassel von 1167 einen 3 tägigen Markt ${ }^{2}$ ). Der Probst des Stiftes leitete daraus für sich die Berechtigung her, die Aufsicht über Maasse nnd Gewichte in der Stadt zu führen, derenwegen es in den 80 und 90 er Jahren des 13. Jahrhunderts $z u$ heftigen Streitigkeiten zwischen dem Stift und der städtischen Verwaltung gekommen ist ${ }^{3}$ ). Der Marktplatz in der Stadt wurde auf erzbischöflichem Grund und Boden errichtet, der ehedem zum Hofe Merhusen gehört hatte ${ }^{4}$ ).

Die wechselvollsten Schicksale hat die Stadt Deut\% während des Mittelalters erlebt. Gegenüber dem mächtigen Köln gelegen, stand es im Theilbesitz der Erzbischöfe von Köln und der Grafen von Berg. ${ }^{5}$ ). Diese ungünstigen Umstände machen die Stadt je nach der politischen Constellation zum beständigen Spielball der drei Interessenten. Schon im Anfange des 12. Jahrhunderts wurde von Deutz aus rheinaufwärts bis nach Coblenz Handel getrieben ${ }^{6}$ ). In Jahre 1230 erlaubt Erzbischof Heinrich von Köln den Bïrgern von Deutz die Stadt zu befestigen und mit Mauern zn umgeben und verheisst ihnen Bedefreiheit ${ }^{7}$ ). Wegen der Deutzer Befestigung aber brachen zwischen dem Errbischof Conrad von Köln, dem Herzog Heinrich von Limburg als Grafen von Berg und der Stadt Köln ernste Zwistigkeiten aus. Um deren Grund fortzuschaffen,

1) Der letztere Termin ist im Deutzer Recht (Grimm, Weisthümer III S. 2) allein aufgeführt. Vgl. dagegen die Uebereinstimmung im Zülpicher Recht unten S. 16 mit den obigen Festsetzungen.

2) Günther, Cod. dipl. Rheno-Mosellanus I 183.

3) Vgl. Urkunden St.-A. Düsseldorf, Bonn-Cassiusstift 38. Mit ihren Ansprüchen gegenüber dem Stift treten auf iudices vid. scultetus et advocatus, scabini, consules, ma gis tri civium ceterique opidani Bunnenses (Urkunde von 1289). Nach der Bulle Papst Nicolaus IV. von 1290 richtet sich die Klage gegen den Schultheissen Weinrich, zwei benannte Schöffen, 3 benannte majores und die Gemeinheit der Stadt Bonn.

4) S. Lacomblets Archiv II S. 299 und 317.

5) S. die Urkunde von 1240, Lacomblet, Urkundenbuch II 249.

6) S. die Coblenzer Rheinzollrolle, K eutgen, Urkunden zur städtischen Verfassungsgeschichte 80.

7) Lacomblet, Ukb. II 166 . 
wurden 1242 die Befestigungsanlagen der Stadt Deutz wieder beseitigt ${ }^{1}$ ), ja selbst die Schutzwebren der Abtei niedergelegt und der Thurm des Klosters zum Theil abgetragen. Die Besitzer von Deutz gingen gegenseitig die Verpflichtung ein, weder in Gebiet, noch in Gerichtshezirk, noch auch in dem Dorf oder dem Kloster Dentz ${ }^{2}$ ) eine Befestignng selbst anzulegen oder deren Anlage zu dulden. Eine ähnliche Verpflichtung binsichtlich Deutz' ubernahm 1257 Graf Adolf von Berg gegenüber der Stadt Köln $\left.{ }^{3}\right)$. Unter Erzbischof Siegfried von Köln scheint dann der Ort zeitweise aufs Neue mit Mauern umgeben gewesen zu sein ${ }^{4}$ ), jedoch im Jahr 1317 fallen alle Anlagen zum Schutze von Deutz abermals dem Widerspruch der Stadt Köln zum Opfer ${ }^{5}$ ). Es half auch nicht viel, dass die Erzbischöfe Engelbert 1364 und Friedrich 1371 den Bürgern von Deutz die städtischen Rechte aufs Nene bestätigten ${ }^{6}$ ). Am Ende des 14. Jahrh. rangiert der Ort unter den Freiheiten, wenngleich die Erinnerung an die ehemalige städtische Herrlichkeit fortlebt ${ }^{7}$.

Das interessante Recht der Stadt Deutz $\left.{ }^{8}\right)$, welches sich als Schöffenweisthum, doch sicher wohl ans dem 13. Jahrbundert, darstellt, zeigt uns als einen wesentlichen Untersehied des Stadtgerichts gegenüber dem Landgericht, dass ersteres nicht auf die drei ungebotenen Dinge beschränkt bleibt. Diese bestehen zwar auch in Deutz unter dem Vorsitz des Vogtes fort, daneben tritt nun aber der Schultheiss als ständiger Richter ${ }^{9}$ ), um den Bürgern eine promptere Rechtspflege zu vermitteln. An dieser Vergünstigung nehmen auch die Bewohner der fünf um Deutz gelegenen Orte

1) Ebenda 274 .

2) a. a. O. nec in villa vel in claustro aut monasterio Tuicrensi.

3) Lacomblet, Ukb. II 444.

4) Kremer, Acad. Beiträge III 177.

5) Lacomblet, Ukb. III 156.

6) St.A. Düsseldorf, Msc. B 2 fol. 204.

7) S. das Weisthum von 1386 bei Lacomblet, Ukb. III 904 und Grimm, Weisthümer III S. 4. „Duytz, dat vurmails eyn sloss ind stat is geweist ind noch hude dis dages eyne vryheit is."

8) Abgedruckt bei Grimm, Weisthümer III S. 1 ff.

9) a. a. O. S. 1. Item officiatus sive scultetus domini Coloniensis per totum annum presidebit iudicio, exceptis tribus temporibus ut supra dictum est. Bezug genornmen wird hiermit auf die tria iudicia non indicta. 
Die Entstehnug der Städte des Erzstifts Köln am Nierlerrhein, 11

einen gewissen Antheil ${ }^{1}$ ). Ich muss darauf verzichten hier die Einzelparagraphen des Deutzer Stadtrechtes näher zu erörtern; hervorgehoben sei nur noch, dass kein Bürger den andern ohne gegründete Ursache vor Gericht laden oder zum Zweikampf fordern durfte.

Die Periode der Städteerhebungen im Niederstift Köln wird dadurch eröffnet, dass Mitte Juli 1228 Erzbischof Heinrich den Burgern von Rees und Xanten die Erlaubniss ertheilt, ibre Wohnorte zu befestigen ${ }^{2}$ ). In Rees und Xanten waren seit alter Zeit kirchliche Niederlassungen. Auch sehen wir dort bereits 1142 Kaufleute angesiedelt, welehe nit Wesel, Emmerich und anderen Orten des Niederrbems in regem Verkehr stehen ${ }^{3}$ ). Aber erst 1228 , nachdem sie befestigt worden waren, zählten Rees und Xanten zu den Städten. An beiden Orten stellen der Stadtherr, der Er\%bischof von Köln und die kirchlichen Stiftungen Wortstätten für Einwanderer zur Verfügung ${ }^{4}$ ). Für Rees wie für Xanten wurde ein eigenes Gericht konstituirt. Bei ersterer Stadt geschah das in der Weise, dass Erzbischof Conrad von Köln 1246 die Reeser Bürger von der Folge an die Dingstätten in Niedermörmter und Aspel befreite $^{5}$ ). In Xanten bildete das Gericht, wie wir aus einer Urkunde von 1250 erfahren, die Stadt mit ihrem Nachbargebiet (confinium) oder Bezirk (districtus ${ }^{6}$ ). Was die Stadtherrn, den Erzbischof Friedrich von Köln und den Grafen Adolf von Cleve 13927) veranlasste diesen neu zu umgrenzen, wissen wir nicht. Genug, es wurde von dem äussersten Graben der Stadt ab gerechnet in deren Umkreis 400 Fuss tief durch Zeichen, Grenzsteine, ein Gebiet abgesteckt, in welchem der Burgfrieden gelten sollte ${ }^{8}$ ).

1) Sie waren, wie es scheint alle in dem forestum, quod dicitur byvancg gelegen. Vgl. Grimm a. a. O. S. 2.

2) Die Reeser Urkunde ist gedruckt bei Liesegang, Recht und Verfassung von Rees, in der Westdeutschen Zeitschrift, Ergänzungsheft VI S. 100 , die von Xanten bei Binterim und Mooren, Die alte und neue Erzdiözese Köln III 83.

3) Vgl. Liesegang a. a. O. S. 99.

4) Liesegang S. 101 und Binterim und Mooren a. a. O. III 120 und öfters.

5) Liesegang S: 104.

6) Binterim und Mooren III 126.

7) Ebenda IV 402.

8) ... welche burghfrede gaen ind syn sal umb dy stat van 
Hierin war der Antast verboten; nur mit Zustimmung der Stadtherren war es erlaubt, in diesem Berirk sich anzubauen oder Wohnung zn nehmen. Rees und Xanten wurden mit Neusser Recht bewidmet; leider sind uns jedoch aus keiner der beiden Städte Kodifikationen ilıres Mutterrechtes erhalten.

Und auch Rheinberg, das im Februar 1232 (1233) eine mit dem Reeser und Xantener Privileg von 1228 gleichlautende Starlterhebungsurkunde ${ }^{1}$ ) von Erzbischof Heinrich von Köln empfing, hat uns den Wortlaut des Neusser Rechtes nicht überliefert. Wir dürfen mit gutem Grund vermuthen, dass die Stadt von ihrem Schöpfer, dem genannten Erzbischof, neben der Befestigung auch die Einrichtung des "hoen frien fredeloszen gerichts" erhalten hat, welches wir in einer Bestätigungsurkunde Erzbischof Hermanns von Köln ron $1496^{2}$ ) erwähnt finden und worunter wir zweifellos das Schöffengericht zu verstehen haben. Zum Stadtgebiet wurden die Dörfer Winderswyck und die Bauerschaft Berkefort gerechnet ${ }^{3}$ ).

Rees, Xanten und Rheinberg verdanken den Bestrebungen Erzbischof Heinrichs I von Molenark, das zerrissene kölnische Gebiet im Niederstift durch befestigte Orte zu sichern, die Erhebung zu Städten. Sichtlich das gleiche Ziel verfolgte dessen Nachfolger Conrad von Hochstaden mit der Umwandlung des Dorfes Uerdingen zur stadt ${ }^{4}$ ). Die ursprüngliche Anlage erwies sich freilich als nicht günstig gewählt, weil sie den Ueberfluthungen des Rheines zu sehr ansgesetzt war; Erzbischof Siegfried von Köln verpflanzte sie daher etwas landeinwärts vom Rhein. Kirchlich wurde Uerdingen von Budberg losgelöst und die Kapelle daselbst zur Pfarrkirche erhoben. Ferner ward ein besonderes Schöffengericht für die Stadt angeordnet, das deren Bürgern den höheren Frieden verbürgte. Einen Uerdinger Bürger zum Zweikampf herausfordern

Xancten rierhondert vuys lanck van dem urssersten graven, dy nu dar is; ind men sal dem zeychenen mit steynen pelen.

1) Jetzt gedruckt bei Tücking, Geschichte der Stadt Neuss S 349.

2) Vgl. diese Zeitschrift 39 S. 63.

3) Ebenda S. 17.

4) Vgl. F. St ollw erck, Die älteste, bisher unedirte, ... Urkunde über die Erhebung des Ortes Uerdingen zur Stadt durch den Erzbischot von Köln Conrad von Hochstaden. Uerdingen 1876. Die Gründungssowie die Verlegungs-Urkunde sind uns nicht mehr erhalten; wir besitzen nur die Bestätigung der früheren Privilegien durch Erzbischof Heinrich von Virneburg von 1324. 
Dje Entstehung der Städte des Erzstitts Köln am Niederrhein. 1\%

konnte nur derjenige, welcher ein Jahr lang als Bürger in der Stadt gelebt hatte. Den Uerdinger „Buer und Limitengang;" auch "Weidtgang" genannt, lernen wir aus einer Beschreibung des Jahres 1660 kennen $^{1}$ ).

Mit Uerdinger Recht ist $1294^{2}$ ) Kempen bewidmet worden, das als Bollwerk gegen das Gelderland anzusehen ist. Erz bischof Siegfried von Köln erkennt es bei dieser Gelegenheit dankbar an, welche Opfer seine Leute in Kempen gebracht baben, um den Ort nach seinem Gebot mit Befestigungsanlagen zu versehen und damit in eine Stadt umzuwandeln. Er verleiht allen Einwohnern desselben die gleiche Freiheit, welche. die von Uerdingen und von anderen Städten des Erzstiftes geniessen mit der Einscbränkung jedoch, dass die Hörigen der Kirche nicht vollfrei werden, sondern fur den Sterbfall noch zu einer Geldabgabe verpflichtet bleiben. Auch dieses Vorzuges gehen sie verlustig, sobald sle aus der Stadt wieder anf das Land ziehen. Bevorrechtigungent der Kenipener Bürger in Bezug auf den gerichtlichen Beweis durch den Eid sind ebenfalls in das Privileg der Stadt aufgenommen, wie denn auch der Paragraph des Uerdinger Stadtrechtes über den Zweikampf darin eine Stelle hat. Eine Niederschrift von der Stadt Freilıeit und Recht, welche der Stadtsekretär Heinrich tho Wege in der ersten Hälfte des 15. Jahrhunderts besorgt hat, führt an erster Stelle die Strafen auf, welche auf Verletzung des Stadtfriedens gesetzt sind ${ }^{3}$ ).

Kempen bildet schon an Ende des 11. Jahrbunderts ein besonderes Kirchspiel ${ }^{4}$ ). Der Erzbischof hatte daselbst einen Hof. Im Jahre 1188 begegnen wir den cives von Kempen, die jedoch mit den erzbischöflichen Leuten nicht identisch sind ${ }^{5}$ ). Sie erhalten in Jahre 1223 von Erzbischof Heinrich I. von Köln den Triftweg nach dem nahe gelegenen Dorfe Molshoven, das später versehwindet, als Vielıweide \%. Die Schöffen des Landes oder später der Herrlichkeit Kempen, deren Gerichtsbezirk auch die Bezeichnung

1) St.-A. Düsseldorf, Akten Kurköln, Stadt Uerdingen, Hoheitssachen $2 \mathrm{I}$.

2) S. die Urkunde bei Lacomblet, Ukb. IV 677.

3) Vgl. diese Zeitschritt 24, S. 236.

4) S. die Urkunde von 1085 bei L a c o m blet, Ukb. I 238, vgl. auch 280.

5) Binterim und Mooren, Die alte und neue Erzdiözese Köln III 53 6) Ebenda 72. 
"Byvanck" ${ }^{4}$ ) führt, besitzen bereits 1275 ein eignes Siegel ${ }^{2}$. Es hat sich bisher nicht feststellen lassen, ob auch um Kempen ein engerer, Burgbann genannter, Uwkreis gelegt wurde, in welchem der Stadtfriede galt. Die Stadt erhebt im 17. Jahrhundert Anspruch auf die Gerichtsbarkeit auch im Lande Kempen $\left.{ }^{3}\right)$. Städtische Richter finden sich schon 1305 erwähnt ${ }^{4}$ ).

Kempen muss überhaupt schon zur Zeit, als es noch Dorf war, eine stattliche Einwohnerschaft ${ }^{5}$ ) aufzuweisen gehabt haben, denn eben im Jahr der Unwandlung desselben in eive Stadt 1294 erlaubt Erzbischof Siegfried von Köln den Bürgermeistern, Schöffen und der Gesammtheit der Bürger in Kempen durch die Bauermeister von den Amtleuten der Bäcker, Fleischer, Krämer und der ubrigen Aemter in der Stadt, wenn diese ihre Aemter nicht ordnungsmässig führten, eine Busse (Kuhr) zu erheben, welche zur Befestigung und zum allgemeinen Besten der Stadt verwendet werden sollte ${ }^{6}$ ). Demnach sind die Aemter in Kempen erheblich älter als die Stadt.

Die dortselbst angesessenen Ritterbürtigen ${ }^{7}$ ) haben an dem Stadtregiment, wie es scheint, keinen gesonderten Antheil genommen.

Nicht viel später als Uerdingen muss das bis in die Neuzeit hinein zwischen Köln und Jülich strittig gewesene Zülpich in den Rang der Städte eingetreten sein. In einer Urkunde vom 9. September $1251^{8}$ ) führt es den Titel einer Stadt noch nicht; damit, wird es erst $1255^{9}$ ) ausgezeichnet. Die Errbischöfe von Köhn besassen hier von altersher eine Burg $\left.{ }^{10}\right)$. Die Vogtei mit der hohen Gerichtsbarkeit, die Guter Palenz innerhalb Zülpichs und die ausserbalb im Kirchspiel von S. Marien trugen als pfälzisches

1) Vgl, diese Zeitschrift 24 S. 227.

2) Binterim und Mooren a. a. O. III 158.

3) Vgl. St.-A. Düsseldorf, Akten KurköIn, Stadt Kempen Nr. 3.

4) Binterim und Mooren IV 275.

5) Dass auch vor der Erhebung zur Stadt Ansiedler hierhin gezogen sind, beweist eine Urkunde von 1264 bei Binterim und Mooren IV 260.

6) Ebenda III 224.

7) Ebenda IV 275, 320.

8) Lacomblet, Ukb. II 376.

9) Ebenda 410.

10) S. die Urkunde Erzbischof Friedrichs von 1124, Lacomblet, Ukb. I 299. 
Die Entstehung der Städte des Erzstifts Köln am Niederrhein. 15

Lehen die Grafen ron Jülich ${ }^{1}$ ). Ein Schiedsspruch in den Streitigkeiten zwischen Erzbischof Conrad von Köln und dem Grafen Wilhelm von Jülich vom 12. Februar (1254) 1255 stellt fest, dass die Stadt Zülpich (opidum Tulpense), die Burg und was dort ist, ein rechtes Allod der Kölner Kirche seien²). Der Graf von Jiilich su'le zufrieden mit dem sein, was ihm durch das Urtheil der dortigen Schöffen zugewiesen sei. Also im Jahr 1255 besass Zülpich die Eigenschaften einer Stadt, ein Schöffengericht finden wir am Orte. Richarda von Jülich mit ihren Söhnen nahm 1279 die Vogtei von Zilpich in der Stadt selbst und ausserhalb derselben in dem Bereich, welcher durch die vier nächstgelegenen Steine abgegrentt war, vom Erzstift Köln zu Lehen ${ }^{3}$ ). Die Lehensträgerin verzichtete darauf die Bürger von Zülpich vor das Gericht Schivelberg, eine andere Dingstätte des Vogteibezirkes, zu laden und überliess dem Eubischof Siegfried neben anderen alle Rechte innerhalb der Stadt, die ${ }_{n}$ Pelenze $^{6}$ genannt werden. Im Jahre $1288^{4}$ ) erkennen die Richter, Schöffen und die gesammten Bürger von Zülpich in Walram von Jülich und dessen Bruder ihre Herren, soweit sich deren Rechte in der Stadt erstreckten. Der Graf seinerseits gewährleistete in einem Vergleich vom 29. Mär\% (1290) 12915) mit dem zeitigen Erzbischof von Köln aufs Neue das Recht der Bürger der Stadt, das diese von der Folge an einen ausserhalb der Stadt gelegenen Gerichtssitz befreite.

Dank den fortgesetzten streitigkeiten, welche zwischen den Erzbischöfen von Köln und den Grafen von Jülich wegen Zülpichs bestanden, sind wir durch verschiedene Weisthümer ${ }^{6}$ ) über die ziemlich verwickelten Rechtsverhältnisse in der Stadt und deren Unkreis recht gut unterrichtet. Und da jede Partei eifersüchtig darauf hielt, dass ihre alten Gerechtsame gewahrt blieben, haben sich bis in das späte Mittelalter hinein hier Zustände erhalten, welche in ein hohes Alter hinaufreichen müssen und die auch durch das vielfach gleichmachende Stadtrecht nicht aufgehoben sind.

Dieses galt innerhalb des Bezirkes, welcher uns in einem

1) S. die Urkunde von 1209 bei Lacomblet, Ukb. II 27.

2) Lacomblet, Ukb. II 410. 3) Ebenda 730. 4) Ebenda 844. 5) Ebenda 907.

6) Vgl. den Aufsatz von A. Tille, Zum Zülpicher Stadtrecht in dieser Zeitschrift 73 S. 1 ff. 
Weisthum von 1375 als Burgbann ${ }^{1}$ ) vorgeführt wird. Dessen Grenzen sind genau angegeben, sie umfassen das unmittelbar unter den Stadtmanem liegende Gebiet, von dem es 1279 lieisst, dass es durch die 4 nächstgelegenen Steine kenntlich gemacht sei ${ }^{2}$ ). Inmerhalb dieses Burgbannes ${ }^{3}$ ) konnten auch die Schöffen von Zülpich, falls der Amtmaun oder der Fronbote nicht in der Stadt anwesend war, einen Mörder, Dieb, Strassenräuber, Nothziichter oder Mordbrenner dingfest machen. Handelte es sich um eine Verletzung durch offene Wunden, so musste der Amtmann sich uach drei Tagen oder nach 6 Wochen des Thäters versichem und durfte dann keine Bürgen mehr annehmen, es sei denn nut des Herrn und des Klägers IVillen ${ }^{4}$ ). Niemand, das heisst offenbar, kein Bürger durfte in die Haft gesetyt werden, der Bürgen stellen konnte. Wessen Hab und Gut besser war als seine Missethat, bedurfte auch keines Bürgen ${ }^{5}$ ).

Innerhalb des Burgbannes befand sich nun aber noch ein besonderes Burgfriedensgebiet, für welches das Recht des Marktes zn Zulpich galt und das die Parochie St. Peter mit dem Sitz des Hauptgerichts ausmachte ${ }^{6}$ ). Hier haben wir es unverkennbar mit der alten erzbischöflichen Burg und deren Burgbann $/$ thun, der als böherer Friedebezirk in der Stadt selbst bestehen blieb, nachdem der neue Burgbann um die Stadtmauern gelegt war.

Neben dem Hauptgericht bestehen noch zwei andere Gerichte in Zülpich ${ }^{7}$ ), das Palenzgericht, dessen Umfang uns ein Weisthum von $1404^{8}$ ) iiberliefert, ans dem wir ersehen, dass es ausschliesslich Stadtgebiet einschloss, ferner das Gericht Mersburden, zu dem die Orte Bessenich und Sievernich gehörten ${ }^{y}$ ). Dieses

1) Tille a. a. O S. 16 und Lacomblet, Archiv tür die Geschichte des Niederrheins I 253, woselbst das Recht der Stadt Zülpich abgedruckt ist.

2) Lacomblet, Ukb. II 730: extra ipsum opidum infra terninum qui distınguitur per quatuor lapides propinquiores.

3) So haben wir zweifellos die Worte auszulegen: „bynnen der stad van Tzulpg ind enbinnen unser herren gerichte, da wir scheffen zo wysen han. Lacomblets Archiv a. a. O.

1) La comblets Archiv I S. $2 \overline{5} 3$.

5) Fbenda S. 254.

6) Tille a.a. O. S. 16.7 7) Tille a. a. O. S. 10.

8) St.-A. Düsseldorf, Akten Jülich-Berg, Herrschaften 38.

9) Dazu wird noch ein Kramhausergericht genannt, das sich über 
Die Entstehung der Städte des Erzstifts Köln am Niederrhein. 17

bildete das Kirchspiel St. Martin, dessen Kirche ehedem ausserhalb der Stadt rauf den Guden " gelegen haben soll ${ }^{1}$ ). Die Palenz lag in der Parochie St. Marien, za der die Dörfer Geich, Füssenich und Elvenich zählten. Beide Gerichte waren zuständig für die Erbgutter in ibrem Sprengel, desgleichen für alle Gebrechen, welche dieser Güter halber vorfielen, doch vorausgesetzt, dass die Busse nicht höher als 5 Mark abzuschätzen war. Alle bei dem Palenzgericht verhandelten Erbfälle mussten noch an dem nämlichen Tag vor Sonnenuntergang an das hohe Gericht in Zülpich abgeführt werden ${ }^{2}$ ) Mersburden und wabrscheinlich ebenso die Palenz sind alte $\bar{H}$ ofes complexe - Mersburden wird auch "Vogtei" genannt ${ }^{3}$ ) welche bereits vor der Vereinigung mit Zülpich eigene Kirchen oder Kapellen besassen, die dann später in den schutz des Mauerrings der Stadt hinein verlegt wurden. Inwieweit sie in dieser ihre communale Selbstständigkeit bewahrt haben, muss erst noch näher untersucht werden.

Als im Jahre 1436 Erzbischof Dietrich von Köln das Recht der Stadt Zülpich neu niederschreiben liess ${ }^{4}$ ), werden darin wohl Bürgermeister genannt; eines Rathes geschieht jedoch keine Erwähnung.

Als Gruppen von Städten mit gleichen Rechten konnten wir bisher ermitteln Rees, Xanten und Rheinberg, ferner Uerdingen und Kempen, welche sämmtlich wohl durch das uns leider nicht in einer älteren Redaction erhaltene Neusser Recht beeinflusst sind. In der zweiten Hälfte des 13. Jahrb. hat Erzbischof Siegfried von $\mathrm{K} \ddot{\mathrm{l}} \mathrm{h}$ in kurzen Zwischenräumen hintereinander Lechenich und Brühl in Städte umwandeln und ibnen bei dieser Gelegenheit Privilegienbriefe ausstellen lassen, welche in grossen Parthien wortgetreu übereinstimmen ${ }^{5}$ ). Es sind zugleich die ersten ausführlicheren Stadtrechtsverleihungen an Städte des Errstiftes.

Fïssenich und Geich erstreckt haben soll. Vgl. Broix, Erinnerungen an das alte berühmte Tolbiacum, die jetzige Stadt Zülpich $\mathrm{S} 125$ und St.-A. Düsseldort a. a. O.

1) Broix S. 86 .

2) Vgl. St.-A. Düsseldorf a. a. O.

3) Vgl. St.-A. Düsseldort a. a O. Msc. B 2 fol. 133.

4) Abgedruckt in dieser Zeitschrift 62, S. 205-208.

5) Das von Lechenich von 1279 ist in dieser Zeitschrift 62, S. 196 ff. gedruckt. Das Brühler Privileg von 1285 findet man bei Lacomblet, Ukb. II 802 .

Annalen des hist. Vereins LXXIV. 
An oberster Stelle steht die Vergünstigung, dass die Bürger sich 7 Schöffen wählen dürfen, welche jedoch zu den Einwohnern der Stadt zählen miissen. Sie sind, wie auch anderwärts, unabsetzbar. Den Vorsitz in ihren Kollegien führen die erzbischöflichen Schultheissen ${ }^{1}$ ). Die Kompetenz der Gerichte von Lechenich und Brühi ist die von Hochgerichten, vor welchen alle Arten von Strafthaten verhandelt werden.

Die Bürger beider Städte geniessen das Vorrecht, dass sie nicht verhaftet werden dürfen, so lange sie nicht vor den Schöffen der ihnen zur Last gelegı̀en That überführt sind. Einschränkende Bestimmungen zielen daraufhin die Zweikämpfe in den Städten zu vermindern. Ferner darf kein Bürger innerhalb des Bifangs der Städte - bei Lechenich heisst es der Bannmeile oder des Bifangs - durch einen auswärtigen Richter festgenommen werden. Die Beschlagnahme von Bürgergut in diesen Bezirken ist verboten. Klagen gegen Bürger aus denselben sind an den städtischen Richter zu bringen.

Den Einwohnern des Bifangs von Lechenich sowohl wie von Brühl wurde es zur Pflicht gemacht, auf den Glockenschlag hin zur Vertheidigung ihrer Städte mit den Waffen herbeizueilen. Widerspenstige wollte der Stadtherr mit einer Strafe bis zu 5 Mark treffen. Sollten doch beide Anlagen dem Erzbischof Siegfried offenbar dazu dienen, die aufrührerischen Bewohner Kölns besser im Schach halten zu können.

Der Bifang von Brühl unfasste 12 inı Umkreise der Stadt gelegene Dörfer, die in der Urkunde vun 1285 namentlich aufgeführt sind. Daneben besass die Stadt noch einen unmittelbar bei deren Mauern gelegenen „burban“, den wir freilich erst in einer Beschreibung ${ }^{2}$ ) des 17. Jahrhunderts erwähnt finden, in der gesagt ist, dass damals 38 Häuser in ihm standen, während die Stadt deren 133 »ühlte. Wir dürfen diesen „burban“ doch wohl wie bei Uerdingen als den alten Burgbann ansprechen. Den Lechenicher Bifang lernen wir aus einer Urkunde von 1517 näher

1) Hierzu ist auch Oppermann, Kritische Studien zur älteren Kölner Geschichte in der Westdeutschen Zeitschrift 21, S. 23 zu vergleichen. In der Stadt Brühl sind jedoch offenbar nur 7 Schöffen die Urtheilsfinder; darauf weisen auch die $7 \mathrm{Köpfe}$ auf dem Schöffensiegel (Endrulat, Niederrheinische Städtesiegel Taf. VII, 6) hin.

2) St.-A. Düsseldorf, Akten Kurköln, Amt Brühl, Hoheitssachen 5 . 
kennen, welche die Orte Ahrem, Conradsheim, Blesheim, Herrig und Meller als zur Stadt gehörig aufzählt ${ }^{1}$ ).

In jeder der Städte wurde ein Wochenmarkt und ein Jahrmarkt eingerichtet. Während nun aber die Ordnung von Maass and Gewicht und die Ausübung der Lebensmittelpolizei bei Lechenich in den Händen des Schultheissen und zweier Burgmänner lag, welche sie mit dem Beirath der Gemeinde regeln sollten, wurde sie in Brühl durch die Schöffen verwaltet.

Bemerkenswerth ist, dass man in Brühl und Lechenich, wobl um den Mangel einer Stifts- oder Klosterimmunität zu ersetzen, den Wedemhof als Asyl bestimmt hat.

Mit den Anlagen des 13. Jahrhunderts ist die städtebildende Kraft des Erzstifts Köln am Niederrbein eigentlich erschöpft. Denn von den im nächsten Jabrhundert entstandenen Städten Alpen und Linn im Niederstift ${ }^{2}$ ), Ahrweiler, Rheinbach, Linz ${ }^{3}$ ), Unkel und Rhense ${ }^{4}$ ) im Oberstift hat es keine zu nennenswerther Bedeutung gebracht, und wir sind selbst über den Zeitpunkt, wann diese Orte zu Städten geworden sind, nur mangelhaft unterrichtet. Auch die durch Erzbischof Friedrich III. 1373 erfolgte Umwandlung der "villa" Zons in ein "oppidum" bedeutet doch mehr die Verstärkung der Sicherung der dortigen erzbiscböflichen Zollstätte, als dass es gelungen wäre, hier einen belebteren Verkebrspunkt zn schaffen. Die geringe Ausdehnung des uns noch heute erhaltenen Mauerringes von Zons beweist das. Verliehen wurde der neuen Stadt das Recht von Brühl. Neben der Anlage der Befestigung wird die veränderte Stellung, welche Zons in Folge des Gnadenaktes Friedrich III. erhielt, in erster Linie da-

1) Die Urkunde ist zugleich für den engen Zusammenhang dieser Ortschaften mit der Stadt sehr bemerkenswerth (St.A. Düsseldorf, KölnDomkapitel Urk. Nr. 1062). Die burgermeistere, scheffen, raidt ind gantze gemeynde der stadt Lechenich mit yrene in- und zobehoire mit namen Airhem Conreschem, Bledeshem, Harge und Melre verkaufen gemeinsam eine Rente von 3 Gulden.

2) Kaiserswerth, welches im Mittelalter zeltweise zum Erzstift Köln zählte, gehört in den Kreis des Aachener Rechtes.

3) Linz muss beispielsweise 1365 auf die Rathswahl verzichten; der Erzbischof bestellt von nun ab jährlich 13 biedere Männer aus der Stadt und dem Kirchspiel zu einem Rath.

4) Meckenheim erhielt erst im Jahre 1636 durch Erzbischof Fer. dinand"Stadtrecht verliehen (St.-A. Düsseldort, Urk. Kurköln 3200). 
durch charakterisirt, dass es nunmehr einen Gerichts- und Verwaltungsbezirk für sich bildete, der einem in der Stadt residirender Amtmann oder Schultheissen unterstellt war. Die Stadtgemeinde durfte sich mit dem Beirath des Schultheissen jährlich einen neuen Bürgermeister wählen. Die sonstigen Kommunalangelegenheiten, welche in dem kleinen Städtchen zu erledigen waren, haben wohl die Schöffen, wie das ja in den älteren erzbischöflichen Städten anch geschehen 1st, mitbesorgt.

Die Stadtgemeinden im Gebiete des Erzstiftes Köln sind ausnahmslos aus Landgemeinden erwachsen. Diesen Entwicklungsgang dürfen wir wohl auch für Andernach und Neuss ${ }^{1}$ ), von denen er uns nicht ausdrücklich bezeugt ist, voraussetzen. Die Befestigung aber ist es, welche den Ort erst zur Stadt macht. Deutz verliert im Laufe des 13. und 14. Jahrhunderts mehrmals den Charakter der Stadt und gewinnt ihn dann später wieder; der erstere Fall tritt regelmässig ein, so oft ibm seine Mauern und Thürme genommen werden. Bei diesem Wechsel scheinen in den Rechten der Schöffen und der Ortsgenıeinde überhaupt wesentliche Aenderungen nicht erfolgt zu sein.

Die gleiche Bedeutung fur die Erhebung eines Ortes zur Stadt wie die Umwallung hat daneben die Erricbtung eines besonderen Gerichtsbezirkes für diesen. An dem neuen Gericht, und das ist ein wescntlicher Unterschied zwischen Stadtgericht und Landgericht, sind nur am Orte selbst angesessene Persönlichkeiten die Urtheilsfinder und die Angehörigen der Stadtgemeinde haben an diesem ihren ausschliesslichen Gerichtsstand, sie brauchen einer Ladung an eine andere Dingstätte auch derselben Grafschaft oder desselben Vogteibezirkes nicht zu folgen. Das Stadtgericht wird Hochgericht ${ }^{2}$ ). War der Ort bisher bereits eine Hauptgerichtsstätte, so bleiben die drei ungebotenen Dinge des Grafen für diesen in der althergebrachten Weise zunächst bestehen oder aber sie werden, wie wir bei Siegburg sahen, zusammengelegt. Der Schultheiss oder der Richter in der Stadt machte jedoch im 13. Jahrhundert dem Grafengericht erbebliche Konkurrenz, die nm so nachhaltiger wirken musste, als dieser oder sein Vertreter

1) Vgl. doch auch Lacomblets Aufsatz: Die letzten Spuren des fränkischen Salhofes zu Neuss in seinem Archiy II 319 ff.

2) S. das Drühler Stadtrecht von 1285, La comblet, Ukb. II 802. 
Die Entstehung der Städte des Erzstifts Köln am Niederrhein. 21

ständig am Platze anwesend war und jeder Zeit die Hegung des Gerichtes vornehmen konnte ${ }^{1}$ ).

Hören wir nun, dass das Recht, welches am städtischen Gericht ausgeëbt wird, die Bezeichnung Burgrecht führt, dass für zahlreiche kölnische Städte ein unmittelbar um die Mauern der Stadt sich anschliessender Bezirk unter dem Namen Burgbann gefreit ist, dass innerhalb der Mauern der Stadt wie im Burgbann der Burgfriede herrscht und dessen Verletzer schwerer bestraft wird, als es bei gleichen Strafthaten unter anderen Verhältnissen zu geschehen pflegt, so wird man doch geradezu darauf bingedrängt, die Burg als das Vorbild der Städte im Erzstift Köln zu suchen ${ }^{2}$ ). Man darf in diesem Zusammenhang doch dann weiter betonen, dass, wie auf den Siegeln anderer Städte ${ }^{3}$ ), auch auf denen des Erzstifts Bilder von Thoren und Mauern sich fast ausnahmslos vorfinden ${ }^{4}$ ).

Wenn Burgfrieden und Marktfrieden in unserer Ueberlieferung gelegentlich als sinnverwandte Ausdrücke ${ }^{5}$ ) erscheinen, so erklärt sich das nicht bloss aus der gleichartigen lokalen Begrenztheit der beiden Rechtsinstitute, sondern auch daraus, dass die grösseren Burgen überhaupt die rom handeltreibenden Kaufmann gern aufgesuchten Schutzstätten gebildet haben ${ }^{6}$ ). Die Mehrzahl der kölnischen Städte waren Märkte, bevor sie in Städte umgewandelt wurden.

Dass bei dieser Gelegenheit den ortseingesessenen Unfreien

1) S. oben unter Deutz S. 11 .

2) Vgl. Sohm, Die Entstehung des deutschen Städtewesens. Leipzig 1890. Von weiteren Erörterungen über diesen Punkt sehe ich vorläufig ab. Sie können erst zu gesicherten Resultaten führen, wenn man in der Lage ist, das Quellenmaterial zur Geschichte der niederrheinischen Städte insg'esammt zu übersehen. Hier folge nur noch die Bemerkung, dass G. v. Below (Der Ursprung der deutschen Stadtverfassung S. 128) in dem Stadtrecht für Radevormwald von 1400 den Burgbann ebenfalls ermittelt hat. Ferner findet er sich in dem Stadtrecht von Blankenberg aus dem Jahr 1245 bei Gengler, Cod. iur. mun. Germ. S. 236.

3) Vgl. hierzu übrigens v. Below a. a. O. S. 20.

4) S. die Abbildungen bei Endrulat, Niederrheinische Städtesiegel, Taf. VII-IX, Text S. $23 \mathrm{ff}$.

5) So bei Zülpich. Vgl. hierzu Sohm a a. O. S. 18 ff.

6) Das geht z. B. aus einem Burgfirieden für Homburg vor der Mark von 1453 hervor. Vgl. St.-A. Düisseldorf, Akten Jülich-Berg, Grenzsachen Nr. 9. 
Vortheile gewährt wurden, dass für sie anstatt Kurmede und Sterbfall eine Geldabgabe festgesetzt wurde, geschah hauptsäehlich in der Absicht, um sie für die Dienste, welche sie bei dem Mauerbau geleistet hatten, zu entschädigen. Einwanderer traten gegen Zahlung eines Bürgergeldes in den Genuss der städtischen Rechte. Denn darauf war es allgemein auch bei den kölnischen Städten abgesehen, den Berölkerungsstand in ihnen zu erhöhen, um den einzelnen Ort wirthschaftlich zu heben und für den Kriegsfall vertheidigungsfähiger zu machen. Zusammenlegung von früher in einem weiteren Umkreis zerstreut bestehenden Ansiedelungen, die aber in diesen Zustand bereits besondere Gerichtssprengel und Kirchengemeinden waren, lässt sich bei Zülpich nachweisen ${ }^{1}$ ). Vielleicht sind die drei ${ }_{\text {senschappen }}{ }^{2}{ }^{2}$ ) in Rees ähnlichen Ursprungs. Auf die Lage der Pfarrkirchen der Sondergemeinden wird man bei der Untersuchung dieser Frage das Augenmerk richten miissen. Dass beispielsweise bei Bonn die Parrochialkirchen von S. Martin und S. Gangolph so nahe bei der Stiftskirche von St. Cassius und in der äussersten Peripherie des Stadtbildes errichtet wurden ${ }^{3}$ ), ist doch beachtenswerth.

Das Vorhandensein von Sondergemeinden in den kölnischen Städten wird anch durch die im 13. Jahrhundert ziemlich allgemein in der Ueberlieferung der einzelnen Orte bezengten Burmeister bestätigt ${ }^{4}$ ). Bürgermeistern hingegen begegnen wir am frühesten in Xanten $^{5}$ ) und Kempen ${ }^{6}$ ), in den übrigen Städten des Erzstifts Köln jedoch erst in der Mitte des 14. Jahrhunderts 7).

1) S. oben S. 17.

2) Mit dieser Bezeichnung und unter den Namen "Nyestrate", „Rynstrate", „Delstrate", erscheinen sie bereits in Niederschriften des 15. Jahrhunderts in Msc. A $248 \mathrm{im} \mathrm{St.A.} \mathrm{Düsseldorf.} \mathrm{Vgl.} \mathrm{dazu} \mathrm{Liese-}$ gang, Recht und Verfassung von Rees S. 61.

3) Vgl. die ältere topographische Karte von Bonn aus dem 16. Jahrhundert, St.-A. Düsseldorf, Akten Bonn-Cassius 23a. Ueber die verschiedenen villae und Höfe, die vordem anf dem Grund und Boden des späteren Bonn gestanden haben, vgl. Lacomblets Archiv II $297 \mathrm{ff}$.

4) Für Rees vgl. Liesegang a. a. O. S. 63; für Neuss, Tü cking; Geschichte der Stadt Neuss S. 27; für Bonn s. oben S. 9 Anm. 3. Vgl. auch Urkunde von 1290 (Köln-Domstift 342).

5) Im Jahre 1289, Binterim und Mooren, Die alte Erzdiözese Köln III 211. 6) Im Jahre 1294, ebenda 224.

7) In Andernach 1358 s. oben S. 7; in Bonn 1359 bei Lac omblet, Ukb. III 589 . 
Die Entstehung der Städte des Erzstifts Köln am Niederrhein. 23

Der Vermehrung des Geschäftskreises, welche durch die starke Bevölkerungszunahme im 12. Jahrhundert den Schöffenkollegien in Andernach und Neuss erwachsen war, hatte man in dieser Zeit durch die Verdoppelung der Zahl der Mitglieder derselben Rechnung getragen. Neuss ist die erste kölnische Stadt, in der sich eine zweite städtische Behörde, welche neben dem Schöffenkolleg eine bedeutende Stellung einnimmt, nachweisen lïsst. Sie wurde hier durch Erzbischof Conrad von Hochstaden 1259 eingesetzt ${ }^{1}$ ). Die neue Stadtvertretung trägt in Neuss zunächst den Namen des Rathes noch nicht. Erst durch die Bestätigungsurkunde Erzbischof Heinrich II. vom 22. Mai $1310^{2}$ ) erhält sie diesen beigelegt.

Conrad von Hochstaden wollte die Neusser Schöffen und Burger für treue Dienste, welche sie ihm geleistet hatten, belohnen. Zunächst erfuhren die Schöffen seine dankbare Gesinnung, indem er diesen in der fraglichen Urkunde ihr bisheriges Cooptationsrecht neu bestätigte. Der gesammten Bürgerschaft räumte er das Recht ein, von nun ab 12 oder 14 , officiati“, zu deutsch Amtmanner, in der gleichen Zahl, in der das Schöffenkolleg zusammengesetzt war, zu bestellen. Die Amtmänner wurden wie die Schöffen auf Lebenszeit gewählt. Bei nothwendig werdendem Ersatz für einen ausgeschiedenen Amtmann sollte dieser durch die Gemeinheit der Stadt und die überlebenden Amtmänner in einträchtiger Wahl bewerkstelligt werden. Konnte man sich nicht ïber eine geeignete Persönlichkeit einigen, so hatte die Majorität und der vernünftigere Theil der Einwohnerschaft den Ausschlag zu geben.

Die Befugnisse der Amtleute wurden in $\mathrm{der}$ Weise festgelegt, dass das Zeugniss von zweien von ibnen mit der nämlichen Wirkung wie bisher dasjenige von zwei Schöffen zum rechtsgültigen Abschluss von Kauf und Verkauf, ferner von Pfandverschreibungen geniigen sollte, ebenso wie in Gegenwart von zwei Amtmännern eine Schuldklage ihre Erledigung finden konnte. Die richterliche Kompetenz des neuen Rathes, soweit iberhaupt von einer solchen die Rede sein kann, blieb also auf Civilsacben beschränkt. Die hohe Gerichtsbarkeit und die Zeugenschaft in solchen Fällen wurden den Schöffen auch fernerhin vorbehalten,

1) S. die Urkunde bei La comblet, Ukb. II 470.

2) Ebenda III 86. 
und selbst die Beurkundung des Wechsels von Erb und Eigen im Burgbann von Neuss hatte auch für die Zukunft nur durch die Schöffen der Stadt zu erfolgen ${ }^{1}$ ). Zur Festsetzung neuer Statuten für die Stadt, die man "Eininge" und "Kure" nannte, war aber die Zustimnung des erzbischöflichen Schultheissen, der Schöffen, Amtmänner und Burmeister nothwendig, in deren Gesammtheit wir demnach erst das eigentliche Stadtregiment verkörpert sehen müssen.

Wer aber waren die officiati, die Amtmänner, was haben wir unter dem "ambiet", wie es 1310 genannt wird, zu verstehen? Leider spricht sich Erzbischof Heinrich II. von Köln in der Urkunde von $1326^{2}$ ), durch welche er seinem Schultheissen die Befugniss einräumt, die djesem von den Neusser Rathsherren als Amtmänner präsentirten Personen in seinem Namen in ihrem Amte zu bestätigen, nicht näber über deren Funktionen aus. Es liegt nahe als ihre Vorbilder die Amtleute der Kölner Sondergemeinden, die gewesenen Meister derselben anzusehen ${ }^{3}$ ), um so mehr als magistri civium in Neuss ja eben in der Einsetzungsurkunde des Amtmännerkollegs von 1259 erwähnt werden. Daneben müssen wir freilich der anderen Auslegung, welche die Amtmänner fưr Vorsteher der Aemter von Handwerkern und Gewerbetreibenden erklärt ${ }^{4}$ ), die gleiche Berechtıgung zugestehen. „Officiati“ heissen in niederrheinischen Urkunden der zweiten Hälfte des 13. Jahrhunderts auch diejenigen, welche an der Spitze von Berufsgenossenschaften stehen ${ }^{5}$ ). Vielleicht liefern uns bisher noch nicht veröffentlichte Urkunden das Material zur Entscheidung dieser Frage.

Nicht viel besser als mit den „officiati" in Neuss sind wir mit den „majores" daran, aus denen im Jahr 1285 (1286?) in Bonn das

1) Vgl. das Privileg Kaiser Friedrichs III. von 1475; Tücking, Geschichte der Stadt Neuss 359.

2) Tücking, Geschichte der Stadt Neuss S. 349.

3) So Liesegang, Recht und Verfassung von Rees S. 40 . Ueber die Kölner Amtleute vgl. La u, Entwicklung der kommunalen Verfassung und Verwaltung der Stadt Köln S. $164 \mathrm{ff}$.

4) Tücking, Geschichte der Stadt Neuss S. 26.

5) So ist 1294 von den pistoribus, carnificibus, tabernariis ceterisque officiatis in Kempen die Rede, die in Busse genommen werden sollen, si officia sua, quibus prefuerunt, minus debite rexerunt. Binterim und Mooren, Die alte Erzdiözese Köln III 224. 
Die Entstehung der Städte des Erzstifts Iiöln am Niederrhein. 25

Rathskolleg seinen Ursprung genommen hat ${ }^{1}$ ). Sie werden bei dieser Gelegenheit uns zun ersten Mal genannt und wir finden einge von ibnen 1290 in einer Bulle Papst Nikolaus IV. ${ }^{*}$ ) namentlich aufgefübrt; seitdem verschwinden sie wieder.

Für Bonn hatte Erzbischof Conrad von Köln noch 1244 bestimmt, dass auch die Verwaltung in der Stadt durch die Schöffen besorgt werden sollte, dass Bonns Frenheiten, Rechte und achtungswerthe Gewohnheiten nach Schöffenurtheil zu ändern und $\mathrm{zu}$ bessern seien. Vierzig Jahre später lernen wir hier einen Ausschuss von angeseheneren Bürgern (majores) kennen, der mit der Gemeinheit im Gegensat\% zu den Schöffen steht, welche die städtische Bede von ilurem Besitz zu zahlen sich weigerten. Das Schöffenkolleg zog nicht nur den Kürzeren in diesem Streite, es musste es sich auch gefallen lassen, dass ihm mit Genehmigung Erzbischof Siegfrieds 1285 (1286?) eine Behörde zur Seite gesetzt wurde, welcher dem Wortlaute der darüber verfassten Urkunde zufolge das Stadtregiment und die Wabrung der städtischen Rechte und Freiheiten eigentlich ausschliesslich übertragen wurde. Motivirt ist die neue Institution mit dem täglichen Anwachsen der Bevölkerung. Die opidani majores universitatis erhielten die Befugniss zwölf Personen oder auch eine geringere Anzahl (legales et fidedignas infra opidurn) za wählen, welche der Stadt besser vorzustehen, deren Interesse mit mehr Wärme wahrzunehmen und den Handelsverkehr ordnungsmässiger zu regeln in Stande wären; wir müssen doch dazu ergänzen, nals es die Schöffen bisher gethan hatten". Was aber dieser Rath zum Woble der Stadt anordnen würde, dem sollte die Gemeinheit nachkommen. Das Vorgehen gegen Zuwiderhandelnde behielt sich der Erzbischof selbst vor. Die Spezialbefugnisse des Rathes werden dann in ähnlicher Weise näher umgrenzt, wie wir das bei dem Neusser Rath gesehen haben: das Zeugniss der Zwölfer oder Zweier von ihnen galt in allen Civilsachen als unbezweifelbare richterliche Entscheidung, nach der Sehöffen und Richter ihr Urtheil fällen mussten. Auch die Marktpolizei ist in Bonn Sache des Rathes, wie wir aus der Rechtsaufzeichnung des 14. Jahrhunderts erfahren; die Strafbestimmungen für Vergehen solcher Art setzt der Rath fest, der Straf-

1) S. die Urkunde bei Lacomblet, Ukb. II 799.

2) St-A. Düsseldorf, Urkunden Bonn-Cassius Nr. 38. 
26 Th. Ilgen: Die Entstehung der Sticlte des Erzstifts Köln etc.

vollzug liegt jedoch auch hierbei wieder in den Händen des Schultheissen und der Schöffen.

Den „majoribus" von 1285 war es anheimgegeben, nach Jahresfrist den Rath ganz oder theilweise zu erneuern. Der im genannten Jahre zum ersten Mal bestellte Rath, sowie alle jährlich neu gewählten Mitglieder mussten dem Erzbischof den Treueid leisten.

Ueber das Aufkommen des Rathes in den anderen kölnischen Städten, in Rees, Kempen, und Rheinberg haben wir aus dem Ende des 13. und dem Anfang des 14. Jahrhunderts zwar Nachrichten, sie geben uns aber über dessen Ursprung und Geschäftskreis keine Auskunft. Wir können daher nur vermuthen, dass auch an diesen Orten, wie in Neuss und Bonn, das Zeuynissvermögen in Civilsachen zu den frühesten Kompetenzen des Rathes gezählt hat. Es ist einleuchtend, dass der Kaufmannsund Handwerkerstand, denen auch die Städte des Erzstiftes Köln ibre Blütheperiode verdanken, ein ganz hervorragendes Interesse daran haben mussten, in solchen Fällen geschäftlichen Verkehrs wie bei Käufen und Verkäufen, bei Schuldklagen ebenfalls ein entscheidendes Wort mitreden zu dürfen. Vermöchten wir die Amtmänner in Neuss, die "majores" in Bonn nach stand und Beruf sicher einzuordnen, so wiurden wir ja viel klarer in diesen Verhältıissen sehen. Somit aber müssen wir uns an der Thatsache genügen lassen, dass der Rath bei seinem Auftreten in das Geschäftsgebiet der Schöffen übergreift, die bald gutwillig, bald gezwungen ibm ebenfalls ein Plätzchen an der Gerichtsbank liberlassen. Dass der Rath je nach der Gunst der Zeitumstände seine Befugnisse erweitert hat, wird man, da die Bevölkerungsschicht auf welche er sich stützte, nach irgend einer Richtung hin zu Ansehen gelangt ist, als den naturgemässen Gang der Entwicklung bezeichnen miissen. In wie weit ihm das in den einzelnen Städten unseres Gebietes gelungen ist, das nachzuweisen, wird die Aufgabe eingehenderer Quellenstudien werden müssen, die gerade am Niederrhein noch zu ganz beachtenswerthen Erträgnissen führen dürften. Bürgermeister und Rath sind hier freilich nicht die fur die Entstehung des' Städtewesens Ausschlag gebenden Faktoren.

1) S. Lacomblets Archiv II 317 und 319. 